\title{
DOENÇA DE MADELUNG (LIPOMATOSE CERVICAL BENIGNA SIMÉTRICA): RELATO DE DOIS CASOS*
}

\author{
Ricardo Pires de Souza ${ }^{1}$, Ademar José de Oliveira Paes Junior² ${ }^{2}$ Abrão Rapoport ${ }^{3}$
}

Resumo Os autores relatam dois casos da doença de Madelung, que consiste em depósitos simétricos de gordura localizados no pescoço, ombros e mediastino superior, envolvendo nervos e vasos. Recomenda-se o uso da tomografia computadorizada, a qual confirma a natureza lipóide da massa, útil no estadiamento pré-operatório e acompanhamento pós-cirúrgico.

Unitermos: Doença de Madelung; Lipomatose cervical; Tomografia computadorizada.

Abstract Madelung's disease (symmetric benign cervical lipomatosis): report of two cases.

The authors report two cases of patients with Madelung's disease, a symmetric fat deposit in the neck and superior mediastinum that involves nerves and vessels. Computed tomography can reveal the fat contents of these masses and guide preoperative staging and post-surgical follow-up.

Key words: Madelung's disease; Cervical lipomatosis; Computed tomography.

\section{INTRODUÇÃO}

A lipomatose cervical benigna simétrica (LCBS) é afecção rara, de etiologia desconhecida $^{(\mathbf{1})}$. Foi mencionada na literatura pela primeira vez por Brodie em 1846, e uma série de 33 casos foi descrita por Madelung em 1888. Dez anos mais tarde, Launois-Bensaude descreveu 65 casos semelhantes, que foram chamados de adenolipomatose múltipla simétrica ${ }^{(2)}$.

\section{RELATO DOS CASOS}

Caso 1 - Homem de 47 anos de idade, pardo, natural de Pernambuco, referindo tumores indolores em ambas as fossas supraclaviculares, que tiveram aumento progressivo num período de seis anos. Era diabético, em tratamento com hipoglicemiante oral, e tabagista há 20 anos. Não relatava história de etilismo. O exame físico evidenciou, à palpação, lesões nodulares, profundas, de consistência gordurosa, em fossas supraclaviculares e regiões cervical,

* Trabalho realizado no Complexo Hospitalar Heliópolis, São Paulo, SP.

1. Diretor do Serviço de Diagnóstico por Imagem do Complexo Hospitalar Heliópolis.

2. Médico Residente do Serviço de Diagnóstico por Imagem do Complexo Hospitalar Heliópolis.

3. Coordenador do Curso de Pós-Graduação em Cirurgia de Cabeça e Pescoço do Complexo Hospitalar Heliópolis.

Endereço para correspondência: Dr. Ricardo Pires de Souza. Avenida Aratãs, 78, apto. 22, Moema. São Paulo, SP, 04081 000. E-mail: ricapires@ig.com.br

Recebido para publicação em 27/9/2002. Aceito, após revisão, em 25/11/2002. parotídea-masseterina e submandibular, bilateralmente. A rinoscopia e a oroscopia não evidenciavam sinais de lesões.

Foi realizada tomografia computadorizada (TC), que evidenciou massa com densidade de gordura na região cervical, estendendo-se até as regiões supraclaviculares (Figura 1).

Após levantada a hipótese diagnóstica de doença de Madelung, foi realizada ressecção parcial de massa gordurosa. A histologia mostrou achados compatíveis com LCBS.

Caso 2 - Homem de 35 anos de idade, branco, etilista, com história de crescimento progressivo de massas cervicais bilaterais, indolores. $\mathrm{O}$ exame físico sugeria a presença de nódulos de consistência gordurosa na região cervical, bilateralmente. O exame laringoscópico não evidenciou sinais de lesão.

A TC confirmou a natureza gordurosa das massas (Figura 2). Foi realizada excisão cirúrgica de caráter estético.

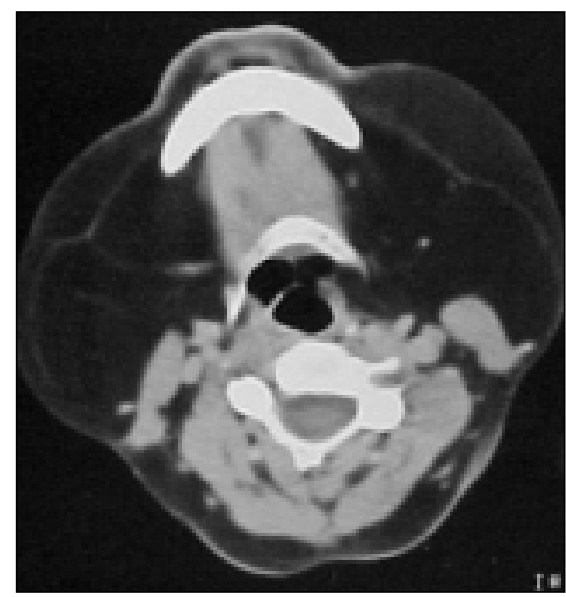

Figura 1. Tomografia computadorizada axial. Acentuado aumento de tecido gorduroso na região anterior do pescoço.
Figura 2. Tomografia computadorizada axial. Obsenvar a medida do coeficiente de atenuação, igual a $-105 \mathrm{UH}$.

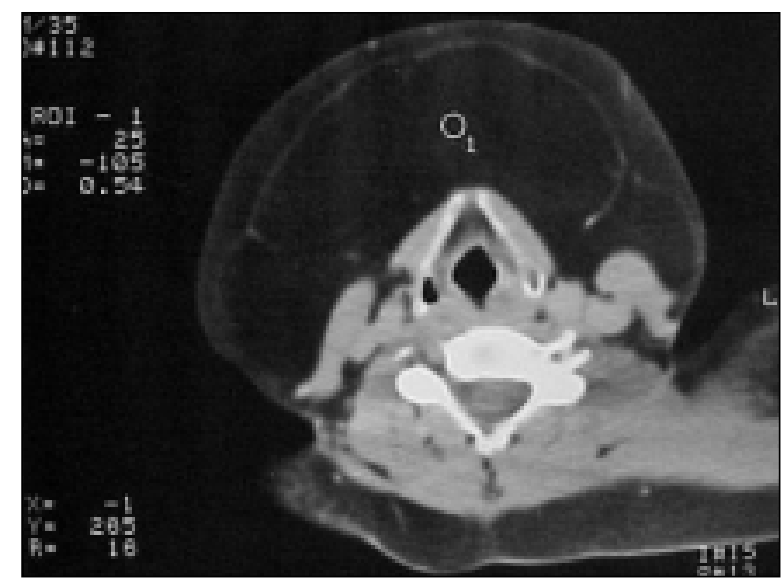




\section{DISCUSSÃO}

A LCBS consiste em depósitos simétricos de gordura, localizados no pescoço, ombros e mediastino superior. Este tecido lipomatoso distribui-se difusamente no compartimento subcutâneo, entre os planos musculares, envolvendo nervos e vasos ${ }^{(3,4)}$. O caráter difuso das lesões e o fato de se tratarem de acúmulos gordurosos faz com que a ultra-sonografia tenha dificuldade de avaliá-las. A principal complicação é a compressão laríngea, podendo levar, muito raramente, a insuficiência respiratória ${ }^{(5)}$.

A LCBS acomete mais freqüentemente indivíduos do sexo masculino, de origem mediterrânea $^{(\boldsymbol{6})}$, na faixa etária de 30 a 50 anos, estando associada freqüentemente ao etilismo ${ }^{(2)}$. Nos últimos anos foram relatados vários casos salientando a associação da LCBS com anormalidades metabólicas, tais como hiperlipidemia, hiperuricemia intolerância à glicose, acidose tubular renal e anemia macrocítica ${ }^{(5,7-9)}$.

O estudo histológico revela que o tecido gorduroso das massas cervicais é semelhante ao tecido gorduroso normal, exceto pela presença de fibrose intersticial e de depósitos de proteoglicanos ácidos ${ }^{(7)}$.

Inúmeros tratamentos clínicos têm sido propostos, desde dieta e controle metabólico até o uso de salbutamol, sem resultados satisfatórios. O único tratamento que traz melhora temporária, do ponto de vista estético e funcional, é o tratamento ci- rúrgico. Pode ser realizada a exérese clássica ou, mais recentemente, a lipoaspiração ${ }^{(10-12)}$. A lipoaspiração apresenta diversas vantagens em relação à cirurgia convencional, como menor risco de lesão neurovascular e de hematomas pós-operatórios. Além disso, a lipossucção pode ser repetida quantas vezes for necessário ${ }^{(11,13)}$.

A TC é considerada hoje o método de escolha para diagnóstico, estadiamento pré-operatório e acompanhamento póscirúrgico dos doentes na LCBS. Os achados na ressonância magnética (RM) quase sempre se sobrepõem aos da TC, devendose ressaltar que a RM é mais cara e menos acessível $^{(3,14,15)}$. A documentação tomográfica permite afastar os diagnósticos diferenciais de linfoma e de metástases linfonodais. A TC possibilita ainda o estadiamento nos casos em que a LCBS esteja associada a doença maligna, especialmente o carcinoma de células escamosas das vias aéreas superiores, devido ao etilismo e ao tabagismo geralmente associados ${ }^{(\mathbf{9})}$.

Nos casos em que o tratamento cirúrgico se impõe, seja por questões estéticas ou por complicações, a TC informa sobre a extensão e a localização precisa das massas gordurosas, facilitando o planejamento cirúrgico $^{(14)}$.

\section{REFERÊNCIAS}

1. Smith PD, Stadelmann WK, Wassermann RJ, Kearney RE. Benign symmetric lipomatosis (Madelung's disease). Ann Plast Surg 1998;41:671-3.

2. John DG, Fung HK, van Hasselt CA, King WW. Multiple symmetrical lipomatosis in the neck. Eur
Arch Otorhinolaryngol 1992;249:277-8.

3. Ahuja AT, King AD, Chan ES, et al. Madelung disease: distribuition of cervical fat and preoperative findings at sonography, MR and CT. AJNR 1998; 19:707-10.

4. Gabriel YA, Chew DK, Wedderburn RV. Multiple symmetrical lipomatosis (Madelung's disease). Surgery 2001;129:117-8

5. Enzi G. Multiple symmetric lipomatosis: an updated clinical report. Medicine 1984;63:56-64.

6. Feliciani C, Amerio P. Images in clinical medicine. Madelung's disease: inherited from an ancient Mediterranean population? N Engl J Med 1999; 340:1481.

7. BenMosbah T, Hermanns JF, Lother Y, Pierard GE. Lipomatose cervicale de Launois-Bensaude Madelung. Tun Méd 1990;68:231-4.

8. Greene ML, Glueck CJ, Fujimoto WY, Seegmiller JE. Benign symmetric lipomatosis (Launois-Bensaude adenolipomatosis) with gout and hyperlipoproteinemia. Am J Med 1970;48:239-46.

9. Herrmann WP, Aulepp H, Huffmann G. Lipomatosis symmetrica benigna mit Hiperlipoproteinamie Typ IV und Epipharix-Karzinom. Z Hautkr 1978; 53:580-6.

10. Carlsen A, Thomsen M. Different clinical types of lipomatosis. Case report. Scand J Plast Reconstr Surg 1978;12:75-9.

11. Samdal F, Kleppe G, Tonvang G. Benign symmetric lipomatosis of the neck treated by liposuction. Scand J Plast Reconstr Surg Hand Surg 1991;25: 281-4.

12. Granato L, Eckley C, Takara C, Lancellotti CL. Lipomatose benigna simétrica. Doença de Madelung - relato de dois casos. F Méd 1994;109:1-5.

13. Faga A, Valdatta LA, Thione A, Buoro M. Ultrasound assisted liposuction for the palliative treatment of Madelung's disease: a case report. Aesthetic Plast Surg 2001;25:181-3.

14. Enzi G, Biondetti PR, Fiore D, Mazzoleni F. Computed tomography of deep fat masses in multiple symmetrical lipomatosis. Radiology 1982;144: 121-4.

15. Watt AJ, McMillan N. Multiple symmetric lipomatosis - MR appearances. Clin Radiol 1999;54:77880. 